\title{
La estimulación de la actividad de estudio en el proceso pedagógico en la secundaria básica: una responsabilidad compartida
}

\section{The stimulation of study activity in the pedagogical process in the basic secondary school: a shared responsibility}

\author{
Tamara de Jesús Martínez Abreu ${ }^{1}$ \\ epp1516@ucf.edu.cu \\ https://orcid.org/0000-0002-5240-4758 \\ Dr. C. María Magdalena López Rodríguez del Rey² \\ mmrodriguez@ucf.edu.cu. \\ https://orcid.org/ 0000-0002-3425-4792 \\ Lic. Meibys Laura Ravelo Valdés ${ }^{3}$ \\ mlravelo@ucf.edu.cu \\ https://orcid.org/0000-0002-4650-6432
}

Recibido: 1/06/ 2020, Aceptado: 1/09/2020

\section{RESUMEN}

El análisis de las posiciones y consideraciones acerca de la actividad de estudio en el proceso pedagógico permite identificar el valor que se le concede a la unidad de acción entre los diferentes actores educativos. Sin embargo, la carencia de una clara definición de sus funciones en torno a esta temática se considera un factor que influye en que aún no se logre una proyección adecuada de las intervenciones pedagógicas, psicológicas y didácticas que pueden hacer para estimular la actividad de estudio. Este estudio realiza una investigación documental analítica con el objetivo de aportar conceptual y metodológicamente en las transformaciones educativas y abrir el debate respecto al tema. Se concluye que en el proceso pedagógico de Secundaria Básica la actividad de estudio debe ser consciente, deliberada, requiere tiempo y esfuerzo, pues está orientado al logro de metas específicas. Se evidencian necesidades de recursos, falta de preparación y tiempo en el proceso pedagógico para la estimulación de los alumnos para la actividad de estudio. Las propuestas realizadas posibilitan la estimulación de los alumnos toman en cuenta los requisitos teóricos y metodológicos y se distinguen por el papel que se le otorga a padres, docentes y psicopedagogos para modificar la disposición de los alumnos hacia el estudio.

Palabras clave: estimulación, actividad de estudio, proceso pedagógico, secundaria básica

\footnotetext{
${ }^{1}$ Estudiante de 5to año. Pedagogía Psicología. Universidad de Cienfuegos "Carlos Rafael Rodríguez". Cienfuegos, Cuba.

2 Profesora Universidad de Cienfuegos "Carlos Rafael Rodríguez". Cienfuegos, Cuba.

3 Profesora Universidad de Cienfuegos "Carlos Rafael Rodríguez". Cienfuegos, Cuba.
} 


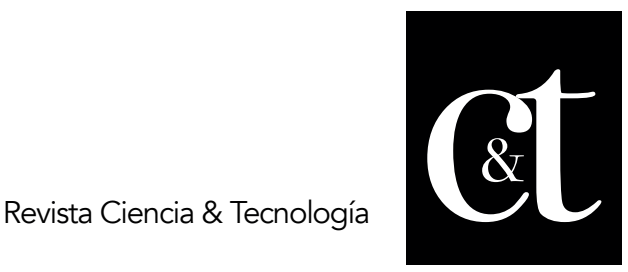

No. 28, 31 de octubre de 2020

ISSN impreso: 1390 - 6321

ISSN online: 2661 - 6734

\begin{abstract}
The analysis of the positions and considerations about the study activity in the pedagogical process allows to identify the value that is granted by the unit of action among the different educational actors. However, the lack of a clear definition of its functions around this theme is considered a factor that has an influence on the fact that an adequate projection of the pedagogical, psychological and didactic interventions that can be done to stimulate the study activity is not yet achieved. This study aims to contribute conceptually and methodologically to educational transformations and open the debate on the subject. It is concluded that in the pedagogical process of Basic Secondary the study activity must be conscious, deliberate, requires time and effort, since it is oriented to the achievement of specific goals. On the other hand, resource needs, lack of preparation and time are evident in the pedagogical process for the stimulation of the students for the study activity. The proposals made allow the stimulation of the students, taking into account the theoretical and methodological requirements and are distinguished by the role that is given to parents, teachers and psycho pedagogues to modify the disposition of students towards study.
\end{abstract}

Keywords: stimulation, study activity, pedagogical process, Basic Secondary

\title{
Introducción
}

Las reflexiones acerca de la actividad de estudio en los resultados del proceso pedagógico, enfatizan en reconocer la importancia que esta tiene, sobre todo, en el éxito académico, lo que ha llevado a identificarse como un factor que determina en el futuro desempeño y a lo largo de la vida. Desde este referente se establece que dentro de los diferentes niveles de enseñanza la actividad de estudio deberá incluirse en las estrategias de formación integral que se llevan a cabo en las escuelas como elemento básico para obtener resultados favorables en el aprendizaje de los estudiantes.

En este propósito se fundamentan diferentes alternativas para orientar a los estudiantes en esta actividad y aun cuando sus propuestas tienden a privilegiar un aspecto más que otro, existe consenso en la importancia del estudio individual y de las actividades grupales, la necesidad de utilizar técnicas de estudio según el nivel educativo, diversificar las actividades a partir del diagnóstico de habilidades, considerar como un factor determinante a la motivación Bermúdez (2002).

Sin embargo, los informes de calidad educativa que se realizan a nivel mundial, en las últimas décadas advierten que en general se aprecia que cada vez más disminuye el tiempo que se dedica a la actividad de estudio y que este comportamiento está asociado directamente al ascenso progresivo por los diferentes niveles educativos.

Así, en las concepciones acerca de la actividad de estudio existen tendencias que van desde las posiciones que consideran que es la actividad que realizan en la escuela hasta limitar su alcance a la resolución de las tareas que el maestro indica (tarea para la casa). Esta concepción limita el propósito de este tipo de actividad y que tiende a afectar el desarrollo de habilidades para enfrentar el estudio como actividad permanente y desarrolladora que permite ampliar, sistematizar y enriquecer conocimientos y habilidades al mismo tiempo que forma actitudes y valores asociadas 


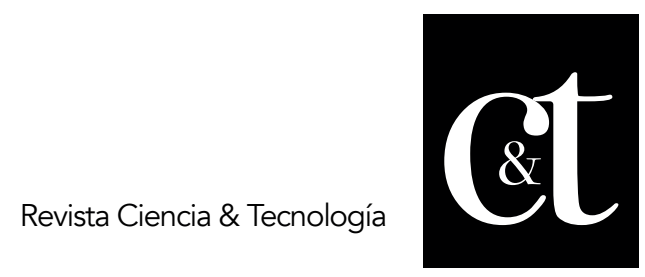

No. 28,31 de octubre de 2020

ISSN impreso: 1390 - 6321

ISSN online: 2661 - 6734

a la responsabilidad, la constancia, el esfuerzo, la voluntad y la creatividad (Collazo, 2001).

En las Secundarias Básicas, el modelo pedagógico (Modelo de Escuela, 2008) establece que la actividad de estudio es la actividad fundamental de los alumnos y que en ella descansan las influencias para su formación integral, de cómo enfrenta la actividad de estudio, dependen los resultados que obtiene en el aprendizaje, se configuran las decisiones para la continuidad de sus estudios, el desarrollo del emprendimiento ante la necesidad de solucionar los problemas propios de las diferentes asignaturas y de la vida cotidiana, con una actitud transformadora y valorativa; y desarrollar la motivación ante el estudio individual y colectivo, a partir de la comprensión de su necesidad e importancia para el desarrollo exitoso de las tareas docentes.

Las reflexiones críticas acerca del cumplimento de este tema en los trabajos presentados en los congresos internacionales de Pedagogía en la última década (Alfonso, 2012) y el I Taller internacional de Secundaria Básica en La Habana (2017), coinciden con los análisis que realizan González, García y Reyes (2016) y lo aseveran Gil, Morales y Chou, (2018) en Cienfuegos. Estos autores enfocan el tema desde situaciones comunes y enfatiza en que esta es una problemática que merece atención integral, sobre todo orientada a conseguir la colaboración de los diferentes actores educativos pues la preparación del alumno para la actividad de estudio precisa la acción compartida de los docentes y de la familia para que pueda estimular el desarrollo de este tipo de actividad.

La constatación de esta problemática en la provincia, mediante un proceso de exploración empírica, desarrollado en los cursos 2017 -2018 y la práctica pedagógica de las autoras durante el curso 2018-2019 orientada a este tema, confirmó que, por un lado, existe la necesidad de promover reflexiones y propuestas para conseguir un cambio en la manera que se procede actualmente en las escuelas al orientar cómo desarrollar la actividad de estudio; pero, por otro lado, urge promover ideas para lograr la participación de todos los agentes educativos en este propósito, al considerar que estimular este tipo de actividad exige ampliar el margen de posibilidades para estimular en los alumnos de Secundaria Básica, su motivación e implicación en la actividad de estudio.

La reflexión teórica como base para construir nuevas propuestas en este tema llevó a las autoras a una revisión y elaboración de una posición teórica que puede ayudar a profesores, directivos a priorizar este tema en las escuelas de educación familiar y sobre todo en la intervención orientadora del psicopedagogo escolar para abordar de manera integral el tema. Los fundamentos y propuestas que se han elaborado con este propósito se incluyen en este artículo con el único propósito de ampliar el debate y la toma de conciencia acerca de la responsabilidad que cada uno de los educadores tiene en la solución de este problema.

\section{Desarrollo}

El estudio forma parte de la vida humana, son aquellas actividades o experiencias que se realizan para un mayor provecho en el aprendizaje estudiantil, ante todo es parte del proceso de formación, ya sea para la superación de todos los obstáculos cognitivos, externos e internos, para realizar las tareas necesarias que orienta la escuela que surgen como inquietudes del aprendizaje (Pérez, 2013). 


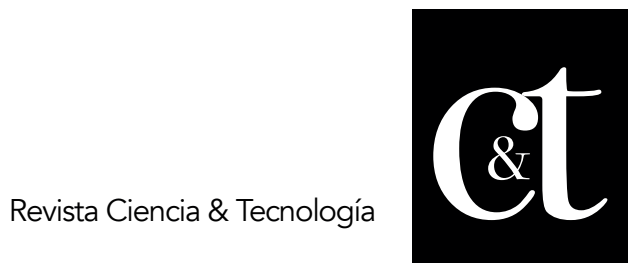

No. 28, 31 de octubre de 2020

ISSN impreso: 1390 - 6321

ISSN online: 2661 - 6734

Da Silva, (2014) dice que la actividad de estudio está regida por un conjunto de habilidades intelectuales y hábitos a través de la cual se intenta adquirir y transformar la cultura; por lo que se convierte en un continuo proceso de aprendizaje. En este mismo propósito, según Covey (1989), el estudio es una actividad esencial en el proceso de crecimiento personal que supone el desarrollo armónico de la inteligencia, la voluntad y la creatividad, para ello se requiere que éstos respondan a las necesidades, capacidades, afectos, actitudes y valores de los alumnos.

Contreras, Cuba, Flores, Salinas y Sulca (2018) afirman que se estudia para adquirir y asimilar conocimientos que permitan desarrollar y organizar mejor la vida de los alumnos. Pero el estudio no solo nos equipa para este logro, sino que es parte de la preparación para la vida. Al mismo tiempo Gil, Morales y Chou, 2018 insisten en que estudiar no es tan sólo repetir de la forma tradicionalista, sino también es hacer otras cosas para que el aprendizaje sea comprensivo y duradero, la alternativa se encuentra en un método de estudio entendido como un proceso continuo de trabajo que empieza en el aula y termina después de las evaluaciones.

Pero, a medio siglo de reflexiones psicológicas y pedagógicas acerca de la actividad de estudio, se identifican como antecedentes teóricos las posiciones que defienden autores como Torroella, (2002); Alfonso, (2012) sus aportes siguen siendo esenciales para comprender este problema y trazar las estrategias necesarias para su estimulación.

En primer lugar, las ideas acerca del carácter individual de los motivos hacia la actividad de estudio; su carácter intrínsecos y extrínsecos y la correspondencia de su manifestación con las características de la personalidad, han sido y siguen siendo condición básica, para asumir el lugar que este tipo de actividad tiene en la formación integral de la personalidad de los alumnos, en lo social, político, ideológico, laboral.

Estos autores, asumen que la actividad de estudio debe ser consciente, deliberada, que requiere tiempo y esfuerzo pues, implica la adquisición de conceptos, hechos, principios, procedimientos y relaciones dentro de un contexto; orientado al logro de las metas que se pretende lograr en un determinado tiempo. Por tanto, como actividad deberá cumplir determinados requisitos psicológicos, pedagógicos y didácticos que favorecen la actividad de estudio.

Desde el punto de vista psicológico, para la actividad de estudio es un requisito contar con una actitud, y el deseo, la disciplina, la persistencia, organización personal, permitirán disponer hacia el cumplimento de las tareas. Pero, el alumno, deberá también encontrarse bien física y mentalmente, asegurar una alimentación sana y una proyección mental positiva que le permita asumir con satisfacción la actividad de estudio diario.

Entre los requisitos pedagógicos es preciso también tener en cuenta los factores ambientales: disponer de un lugar de estudio de uso exclusivo, agradable, ventilado, con el mobiliario adecuado, ayuda a elevar la disposición hacia el estudio. Pero, como requisitos didácticos, es también un aspecto clave que se logre proveer al alumno de una adecuada planificación que inicia con la identificación clara del objetivo, una comprensión detallada de qué debe hacer, dónde puede encontrar las fuentes y las ayudas para lograr la tarea. Así mismo, deberá conocer desde el primer momento, el tiempo del que dispone y las formas en que será evaluada o utilizará el resultado de la actividad de estudio en el proceso pedagógico y su valor a lo largo de su vida. 


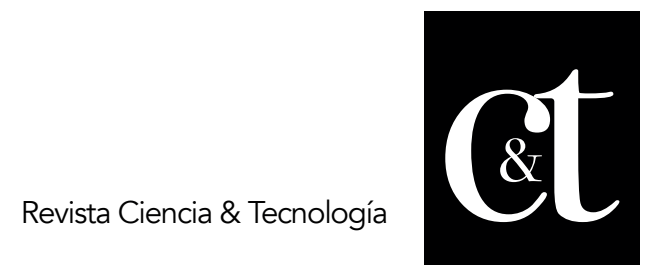

No. 28, 31 de octubre de 2020

ISSN impreso: 1390 - 6321

ISSN online: 2661 - 6734

Luego, cuando se pretende aportar a la estimulación para la actividad de estudio es preciso asegurar estas condiciones y, sobre todo, explorar las necesidades y motivos que tienen los alumnos; pues en ellos se sustenta la orientación hacia la ejecución de dicha actividad. Sin embargo, orientar hacia el cumplimento del deber de estudiar también se basa en diversos motivos que actúan como estímulos donde cada alumno debe internalizar la actividad de estudio como parte de las exigencias de su vida escolar. Estos motivos pueden ser intrínsecos y extrínsecos:

- Los motivos intrínsecos son los de carácter socio personales, vinculado a la percepción y valoración de la importancia que esta actividad tiene en lograr alcanzar los proyectos de vida y necesidades del adolescente: realización, autovaloración que el alumno tiene de sí mismo (motivos personales).

- Los motivos extrínsecos son aquellos que no se corresponden directamente con la esencia para la actividad de estudio, pues constituye un medio para satisfacer otras necesidades, vinculadas a las exigencias de la familia, la sociedad (motivos sociales).

Estos han de verse al mismo nivel o formando parte del mismo grupo de los que responden tanto al desarrollo cognoscitivo y la actividad intelectual como al gusto por el estudio. En este entretejido de motivaciones, es preciso estimular el conocimiento de los métodos y técnicas de estudio las cuales servirán de base para programar la utilización de estas en distintas actividades y materias.

En primer caso, los métodos y técnicas de estudio, que se consideran como recursos para facilitar la actividad de estudio y favorecen el aprendizaje, entre ellos, están:

- Prelectura: es la lectura inicial que le hacemos a un texto para sacar una idea general.

- Notas: es lo que entendemos y anotamos en la hoja.

- Subrayado: es resaltar las palabras que consideremos más importante.

- Esquema: es expresar un texto de manera gráfica o jerárquicamente.

- Resumen: es extraer de un texto la idea central o lo más importante.

- Memorizaciones: grabar los conocimientos que queremos poseer para después recordarlos.

- Lectura analítica: consiste en una lectura detallada y detenida

- Mapas conceptuales: son un medio de visualizar conceptos y relaciones jerárquicas entre ellos.

En efecto, estos métodos y técnicas de estudio podrán ser utilizados en el proceso pedagógico por los alumnos de Secundaria Básica, tanto en actividades curriculares, extracurriculares o en las tareas docentes y extra docentes que se indican a realizar en la escuela, el hogar o en la comunidad. Ellos se presentan como recurso de apoyo y ayuda para que el alumno mantenga el estudio como centro de su actividad. Así los apoyos permitirían que el alumno mantenga la orientación de los objetivos y lo ayuda a facilitar el cumplimento de la tarea (Contreras et al., 2018).

Pero, orientar al alumno para la actividad de estudio debe incluir cierta valoración acerca de los beneficios de tipo espiritual o material que podrá alcanzar. Al respecto se establecen con frecuencia relaciones entre el estudio y sus beneficios: cómo obtener buenas notas, recibir recompensas, autorrealizarse, recibir el reconocimiento de sus compañeros, entre otros, actúan como motivos personales que no deben constituir un fin en sí mismo. Por el contrario, es preciso desarrollar una fuerte 


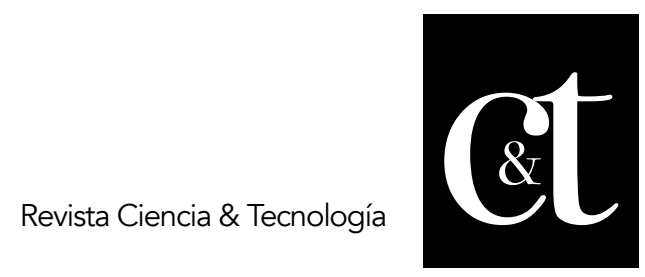

No. 28, 31 de octubre de 2020

ISSN impreso: 1390 - 6321

ISSN online: 2661 - 6734

motivación intrínseca, hacia la propia actividad de estudio; a partir de que ellos puedan apreciar sentimientos de satisfacción hacia las actividades de estudio, los cuales deberán actuar como reguladores del modo de actuación ante las demandas del proceso pedagógico.

En este marco, la búsqueda de información acerca de la estimulación como proceso psicopedagógico se pudo comprender que este término, proviene del latín estimulare, que significa incitar, excitar con viveza a la ejecución de una cosa o avivar una actividad, operación o función (Diccionario de la Lengua Española, 2001).

Pero, este tipo de influencia intencional de carácter psicopedagógico basado en técnicas e instrumentos mediadores que impulsan y dinamizan al sujeto a la acción se define según la naturaleza de las influencias, actúan tanto en las esferas motivacional-afectiva y la cognitivo instrumental de su personalidad, de manera que se compromete al individuo con la ejecución dotando de significación y sentido personal la actividad que realiza.

Se trata de ejercer las influencias educativas que movilizan los recursos personológicos del estudiante, acentúa el desarrollo de conocimientos y habilidades y actitudes que le permiten satisfacer las necesidades de la actividad que puede resultar compleja y demanda impulsar la regulación inductora- ejecutora ante la actividad, hasta que confiere intensidad y dirección (Da Silva, 2014).

Por tanto, la actividad de estudio está vinculada a la esfera motivacional afectiva, cognitiva y metacognitiva, razón por la cual se considera una disposición consciente hacia la ejecución de la actividad, con dominio de habilidades y hábitos que permiten satisfacer necesidades de aprendizaje y obtener resultados personales y sociales. Luego, la estimulación hacia la actividad de estudio, deberá concebirse como un proceso de influencia motivacional, cognoscitiva y metacognitiva, mediado por el educador que permite dinamizar la regulación del estudiante ante la ejecución de las tareas al identificarlas como una necesidad de estudio, padres, familiares, docentes y otros miembros de la comunidad pueden aportar a este propósito.

En este marco las investigaciones de Gil, Morales y Chou, (2018) permiten identificar que al menos existen tres tendencias que caracterizan las creencias y posiciones en la que muchas personas consideran que el alumno que estudia, es porque le gusta y no comprenden la implicación que tienen en esta actividad las influencias orientadoras, reguladoras y de sostenimiento que acompañan el desempeño de la actividad de estudio en las primeras edades. Otro grupo considera que este tipo de actividad se aprende estudiando, sin la orientación y otros aseguran que es la escuela quien tiene que enseñar a estudiar.

En cualquier caso, las posiciones externas conducen a una concepción errada. Es preciso asumir que los adolescentes que estudian son aquellos que han sido expuestos a un proceso de estimulación de motivaciones, conocimientos y actitudes en la que la participación de la familia y docentes resultó imprescindible. Luego, la estimulación del aprendizaje se refiere a una serie de influencias manifiestas en actividades concretas las que se articulan y estructuran para provocar una reacción (interna y externa), para, favorecer un cambio en la apropiación de la experiencia social, que conduce a cambios en los modos de actuación del sujeto con respecto al proceso pedagógico. Por tanto, debe ser concebido como el proceso sistémico de potenciación de las condiciones psicológica y pedagógica del sujeto dirigido al 


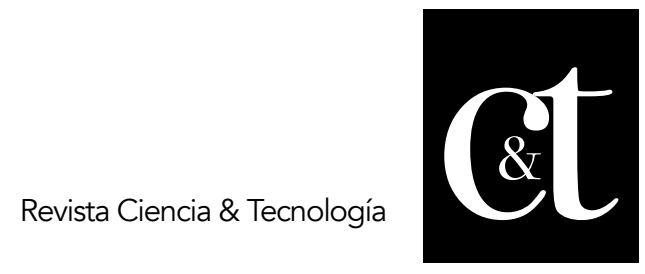

No. 28, 31 de octubre de 2020

ISSN impreso: 1390 - 6321

ISSN online: 2661 - 6734

desarrollo de niveles superiores de autodeterminación en la implicación de los individuos en la actividad de aprendizaje.

Los autores Torroella (2002) y Alfonso (2012), ayudan a comprender que la estimulación del aprendizaje y la motivación para la actividad de estudio deben concebirse como un tipo de influencia psicológica que pueden realizar los diferentes actores educativos y transitan por diferentes grados de complejidad; asumir que la estimulación del aprendizaje se refiere a una serie de influencias manifiestas en actividades concretas las que se articulan y estructuran para provocar una reacción (interna y externa), para, favorecer un cambio en la apropiación de la experiencia social, que conduce a cambios en los modos de actuación del sujeto con respecto al proceso pedagógico.

Desde esta perspectiva, para lograr que el estudio ocupe el centro de atención de los alumnos es necesario comprender que en este tipo de actividad influyen factores pedagógicos, psicológicos y de carácter didáctico.

En el primer grupo, se incluyen aquellos relativos a las condiciones ambientales, organización del lugar, mente y tiempo de estudio. Collazo (2001), señala que en cuanto a organizar el lugar, hace referencia a todas las condiciones físicas del espacio y lugar de donde se pretende estudiar. Organizar la mente, es ordenar el contenido desde lo general a lo particular, puesto que, sin una estructura previa no se puede aprender y organizar el tiempo, es la planificación equilibrada de la jornada diaria y semanal para realizar todos los quehaceres; valorando con racionalidad el alcance de los trabajos o tareas, los períodos libres de descanso, diversión, desarrollo personal, familiar y social, para que armonicen y pueda resultar satisfactoria la actividad a realizar.

En el segundo grupo, que apunta a lo psicológico se refiere a la estimulación de las motivaciones, interés, expectativas y proyectos que tengan los alumnos a partir de la situación de desarrollo actual y potencial que posean y desde los cuales el estudio puede constituir la vía para lograrlo. Así utilizando recursos de apoyo y ayuda en los que para enfrentar la tarea o trabajo de estudio.

En el tercer grupo, referente a lo didáctico implica la estructura de las tareas, los recursos que dispone el alumno y la planificación de la tarea. En síntesis, es determinar qué, cuándo, y dónde se realizan las actividades.

Por tanto, debe ser concebido como el proceso sistémico de potenciación de las condiciones psicológicas y pedagógicas del sujeto dirigido al desarrollo de niveles superiores de autodeterminación en la implicación de los individuos en la actividad de aprendizaje (González, 1989).

Sin embargo, aunque la bibliografía en torno al tema no es específica en cuanto a identificar y clasificar las funciones y tareas que cada educador debe asumir ante la estimulación de los alumnos hacia la actividad de estudio, se asumen las consideraciones teóricas y metodológicas que ofrecen autores como González, García \& Reyes (2016) quienes permiten precisar que en el contexto de la secundaria básica: La familia es el entorno en el que comienza la estimulación de sus hijos hacia el aprendizaje y la actividad de estudio pues los padres y demás miembros constituyen los ejemplos de referencia para marcar el desarrollo cognitivo y afectivo del futuro alumno. Los padres, sobre todo, tienen la oportunidad de guiar a sus hijos para la 


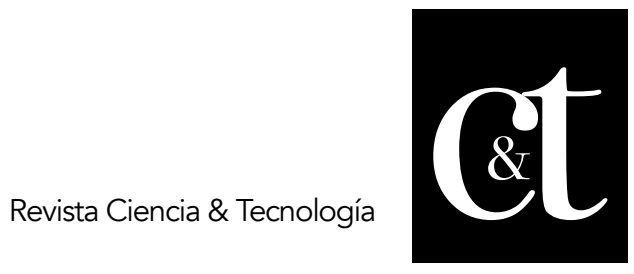

No. 28, 31 de octubre de 2020

ISSN impreso: 1390 - 6321

ISSN online: 2661 - 6734

actividad de estudio preparándolos en la organización de tiempos, espacios, condiciones materiales y ambientales que permitan disponer al alumno para este tipo de actividad.

En este caso, los padres deben mostrar interés y entusiasmo por sus tareas o sus trabajos y manifestar su curiosidad por adquirir nuevos conocimientos, trasmiten a sus hijos esta actitud de forma natural; deben, además, disponer de tiempo libre para enseñarles a buscar la respuesta a sus preguntas, participar en sus actividades con ilusión y demostrar satisfacción cuando consiguen logros significativos. Son algunos aspectos que fomentan la estimulación para la actividad de estudio. Del mismo modo, el ambiente en el hogar también debe incitar al esfuerzo y valorar éste por encima de los resultados, para que sus hijos se sientan estimulados en sus tareas.

Para lograr estas influencias, es necesario que los padres se adecuen a sus capacidades y que la dificultad aumente a medida que adquiere nuevas competencias. Se debe insistir siempre a realizar acciones para las que todavía no está capacitado.

En general, los padres deben comprender que es fácil que sus hijos se desmotiven al comprobar que su esfuerzo no ha valido la pena. Por tanto, es preciso que se les enseñe a superar con esfuerzo y constancia los obstáculos y mostrar confianza en sus aptitudes.

La escuela, por su parte, constituye un espacio de formación, de enseñanza y aprendizaje, donde el alumno encuentre una utilidad presente, pero principalmente futura para su vida. Para lograr que los alumnos encuentren las clases suficientemente interesantes es necesario potenciar su motivación hacia la actividad de estudio. En el ámbito escolar, el docente adquiere un papel primordial.

Para conseguir que los alumnos muestren una disposición positiva para la actividad de estudio, su actitud no se debe limitar a la transmisión de conocimientos, sino que debe poner énfasis en cómo lo hace. Para ello, deberá hacer que sus clases, sean más atractivas y despertar el interés por los contenidos curriculares, de modo que al finalizar se sientan satisfechos de haber aprendido algo, es decir, el interés que plantean los propios alumnos.

En ese caso, los docentes podrán intentar asociar las actividades de la clase con los intereses del estudiante: deportes, música, eventos de actualidad, cultura audiovisual; despertar la curiosidad del estudiante apelando al factor sorpresa; señalar discrepancias existentes entre determinadas creencias de la mayoría de la gente sobre muchos temas y la realidad. También pueden utilizar juegos y actividades físicas, para hacer las clases más divertidas, amables y cercanas a los alumnos.

Introducir variedad en la organización y estructura de las clases para no aburrir a los alumnos con una excesiva monotonía. De esta manera docentes y padres, podrán estimular a los alumnos hacia la actividad de estudio al otorgarles la autonomía y el protagonismo, es hacer a los alumnos sean partícipes en las clases, las intervenciones más importantes que pueden realizar los docentes deben enfocarse a cinco puntos: crear un proyecto personal, despertar interés por la actividad de estudio, fomentar el sentimiento de competencia, mostrar apoyo docente y sentir el apoyo de los compañeros. 


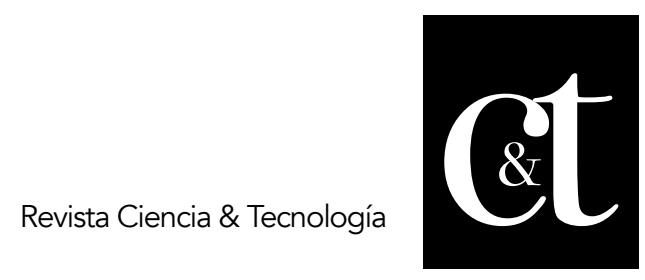

No. 28,31 de octubre de 2020

ISSN impreso: 1390 - 6321

ISSN online: 2661 - 6734

Luego, la adecuación de estas ideas en función de aportar a una concepción pedagógica acerca de la estimulación de la actividad de estudio, se presentan en el cuadro que sigue. Tabla 1. Funciones que se le asignan al docente, familiares y miembros de
la comunidad

\begin{tabular}{|c|c|c|c|}
\hline $\begin{array}{l}\text { Función de } \\
\text { educador / } \\
\text { naturaleza de } \\
\text { la tarea }\end{array}$ & Psicológica & Pedagógica & Didáctica \\
\hline $\begin{array}{l}\text { Padres } \\
\text { familiares }\end{array}$ & $\begin{array}{l}\text { Crear expectativas } \\
\text { de vida } \\
\text { Ejercitarles la } \\
\text { memoria, } \\
\text { concertación }\end{array}$ & $\begin{array}{l}\text { Organizar horarios } \\
\text { Crear espacios propios } \\
\text { adecuados } \\
\text { Asegurar diccionarios } \\
\text { Enciclopedias y otros libros } \\
\text { Disponer un tiempo para } \\
\text { ayudar a resolver tareas } \\
\text { Informarse } \\
\begin{array}{l}\text { Prepararse con el docente los } \\
\text { distractores visuales, los } \\
\text { distractores auditivos y la } \\
\text { desorganización. }\end{array}\end{array}$ & $\begin{array}{l}\text { Proponer temas } \\
\text { curiosos } \\
\text { Solicitarles textos y } \\
\text { materiales de estudio }\end{array}$ \\
\hline Docentes & $\begin{array}{l}\text { Orientar } \\
\text { motivaciones } \\
\text { Explicar importancia } \\
\text { Reconocer las } \\
\text { potencialidades que } \\
\text { poseen, instarlo a } \\
\text { superar barreras } \\
\text { Guía la selección de } \\
\text { métodos } \\
\text { Promover } \\
\text { establezcan que } \\
\text { relaciones entre lo } \\
\text { que estudian y la } \\
\text { actividad de estudio, } \\
\text { la situación personal } \\
\text { y el futuro }\end{array}$ & $\begin{array}{l}\text { Orientar a los padres } \\
\text { Ofrecerles información } \\
\text { Organizar grupos, divulgar las } \\
\text { actividades que realizarán sus } \\
\text { alumnos } \\
\text { Asegurar los apoyos y ayudas } \\
\text { de los demás docentes, } \\
\text { bibliotecarios, profesor de } \\
\text { computación los distractores } \\
\text { visuales, los distractores } \\
\text { auditivos y la desorganización. }\end{array}$ & $\begin{array}{l}\text { Graduar la tarea, } \\
\text { diferenciarla, } \\
\text { individualizarla } \\
\text { contextualizarla } \\
\text { Definirles objetivos, } \\
\text { explicar la importancia } \\
\text { del conocimiento objeto } \\
\text { de estudio en el } \\
\text { aprendizaje del grado o } \\
\text { periodo. } \\
\text { Identificar } \\
\text { complejidades y vías de } \\
\text { resolverlas } \\
\text { Guiar el proceso de } \\
\text { solución de la tarea con } \\
\text { preguntas }\end{array}$ \\
\hline $\begin{array}{l}\text { Miembros de } \\
\text { la comunidad }\end{array}$ & $\begin{array}{l}\text { Reconocer } \\
\text { socialmente a los } \\
\text { alumnos destacados }\end{array}$ & $\begin{array}{l}\text { Crear espacios y actividades en } \\
\text { la comunidad que promuevan el } \\
\text { estudio: concurso, exposiciones } \\
\text { Promover intercambio entre } \\
\text { personas con altos resultados } \\
\text { científicos, culturales, } \\
\text { tecnológicos alcanzados por su } \\
\text { dedicación al estudio y los } \\
\text { alumnos }\end{array}$ & $\begin{array}{l}\text { Promover libros, } \\
\text { materiales informativos } \\
\text { que se puedan utilizar } \\
\text { como medios } \\
\text { (centros y } \\
\text { personalidades } \\
\text { destacadas que pueden } \\
\text { motivar para la } \\
\text { actividad de estudio) }\end{array}$ \\
\hline
\end{tabular}

Fuente: elaboración propia

Desde las ideas antes expuestas se asume como base para estimular la actividad de estudio en el proceso pedagógico en la Secundaria Básica las siguientes consideraciones:

- La estimulación de los alumnos hacia la actividad de estudio en Secundaria Básica asume como un tipo de intervención intencional de carácter psicológico, pedagógico y didáctico que contribuye a la autorregulación gradual del estudiante 


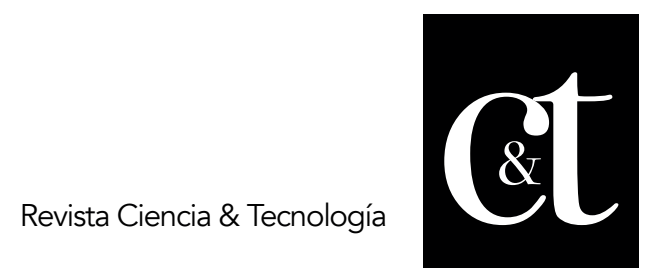

No. 28, 31 de octubre de 2020

ISSN impreso: 1390 - 6321

ISSN online: 2661 - 6734

para el estudio. Desde ellas se logra la influencia intencionada hacia el conocimiento de métodos y técnicas para el desarrollo de una actitud positiva de las actividades de estudio que se orientan y estructuran desde el proceso pedagógico y se desarrollan tanto dentro como fuera de la escuela.

- Es un proceso en que se organizan los estímulos de forma sistemática, se logra dinamizar el desarrollo de habilidades y hábitos que repercuten en el desarrollo a lo largo de la vida. Por tanto, los métodos, técnicas, y condiciones que se crean para este tipo de actividad se convierten en recursos de la estimulación.

- Se concreta en la intervención que realizan docentes, padres, y el psicopedagogo para promover motivaciones, habilidades y hábitos de estudio sistemático provocando una satisfacción en él al comprobar los beneficios y resultados que provienen de la actividad de estudio.

- Se caracteriza por la intencionalidad que las actividades de enseñanza deben poseer para lograr estimular al alumno para que aprenda: primero, a estudiar bajo la influencia directa de una situación externa que lo dispone a estudiar; después, el alumno realiza la tarea bajo la presión indirecta del medio sobre él, pero sustentado en sentimientos y emociones y más tarde pueda estudiar de manera autónoma al conseguir mediante el estudio responder a necesidades, intereses cognoscitivos, sentimientos y convicciones asociados a la importancia del estudio para conseguir proyectos personales y potenciar el desarrollo de su personalidad.

- Es preciso reconocer que por su contenido orientador esta deberá ser desarrollada por un educador (docentes y familiares), los que utilizan algunos recursos psicopedagógicos que movilizan, incitan, provocan y guían la acción del alumno para el estudio a partir de las características de la personalidad de cada alumno y el grupo.

- Exige determinadas condiciones que actúan como estímulos a las circunstancias psíquicas del alumno en función de que estos puedan realizar valoración de sus posibilidades funcionales, habilidades, capacidades imagen que tiene de sí mismo y convertir el estudio en una de las primeras actividades que conforman la jerarquía de necesidades y motivos que movilizan la disposición hacia la actividad produciendo satisfacción y mayores deseos de seguir realizando el estudio.

- La motivación por la actividad de estudio emerge desde estímulos de valoración metacognitiva acerca de necesidades y motivos, hacia la orientación externa de recursos para alumnos y la creación de condiciones que faciliten el desarrollo de las habilidades y hábitos, cerrando el proceso con otras actividades de valoración metacognitiva, donde el sentimiento de satisfacción y el resultado de la actividad constituyen los pilares claves.

Esta posición avala que la estimulación de los adolescentes para la actividad de estudio, se enfocará, primero a estimular motivos, intereses, sentimientos, conocimientos, actitudes proactivas; segundo, impulsar la actividad ejecutora para el estudio y se transformará de manera gradual en una necesidad de realización que se materializa en la autorregulación de la actuación frente a las tareas de aprendizaje, expresándose en la sistematicidad o persistencia, en la estabilidad y la satisfacción del alumno en los diferentes actos que se involucra.

De esta manera, la orientación a la tarea de aprendizaje curricular, para realizarse en la escuela o en el hogar, deber estar cargada de mensajes que favorezcan la formación de disposiciones y motivaciones para el estudio; pero, durante el desarrollo es necesario desplegar estrategias que inciten a actualizar, ampliar, reutilizar, valorar el contenido y el aprendizaje a partir de un sistema para la actividad de estudio. 


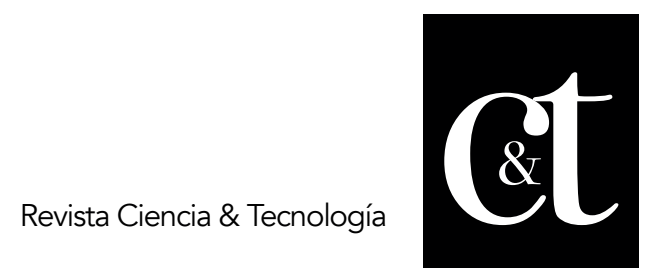

No. 28,31 de octubre de 2020

ISSN impreso: 1390 - 6321

ISSN online: 2661 - 6734

En este marco se entiende entonces que la estimulación posee un carácter afectivo, cognitivo y metacognitivo, de manera que el estímulo que recibe el estudiante es lo que le permite dinamizar el proceso de disposición, dedicación y llegar a desarrollar las habilidades y hábitos que demanda este tipo de actividad. Luego la base del compromiso que contraen los agentes educativos ante la responsabilidad compartida con la estimulación del estudiante de Secundaria Básica hacia la actividad de estudio, se identifica, primero con un cambio en las concepciones de este tipo de actividad y de la manera en que cada uno, desde la función educativa que desarrollan, influyen en que los estudiantes a corto, mediano y largo plazo puedan desarrollar motivos, habilidades para el estudio y para el autoconocimiento ante las exigencias que el estudio presupone.

Cualquier propuesta pedagógica didáctica en este sentido debe asegurar la coordinación desde la dirección de la escuela como condición previa la preparación de todos los agentes educativos, asegurar un clima adecuado y mantener una comunicación y retroalimentación permanente con los participantes en espacios no formales, de manera que las influencias favorezcan las relaciones afectivas entre los actores y el estudiante, creando condiciones para elevar la influencia hacia nuevos objetivos.

\section{Conclusiones}

En la revisión de las concepciones teóricas y metodológicas de estimulación de los alumnos para la actividad de estudio en el proceso pedagógico de Secundaria Básica se pudo determinar que: la actividad de estudio debe ser consciente, deliberada, requiere tiempo y esfuerzo pues implica la adquisición de conceptos, hechos, principios, procedimientos y relaciones dentro de un contexto; orientado al logro de las metas que se pretende lograr en un determinado tiempo. Las necesidades y potencialidades del proceso pedagógico para la estimulación de los alumnos para la actividad de estudio en Secundaria Básica se evidencian en las necesidades de recursos, falta de preparación y tiempo. Las consideraciones que se proponen posibilitan la estimulación de los alumnos para la actividad de estudio en el proceso pedagógico en Secundaria Básica; se tuvo en cuenta los requisitos teóricos y metodológicos y se distinguen por el papel que se le otorga a padres, docentes y psicopedagogos para modificar la disposición de los alumnos hacia el estudio.

\section{Referencias bibliográficas}

Alfonso Moreira, Y. (2012). La participación de la familia y la comunidad en el proceso de enseñanza- aprendizaje en la escuela primaria. Tesis Doctoral, Universidad de Ciencias Pedagógicas "Conrado Benítez García".

Bermúdez, R. (2002). Dinámica de grupo en Educación: su facilitación. Pueblo y Educación.

Collazo B. (2001). La orientación en la actividad pedagógica. La Habana: Pueblo y Educación.

Contreras, V.; Cuba, V.; Flores, G.; Salinas, P. y Sulca, M. (2018). Métodos de estudio. Material didáctico. Universidad de San Martin de Porres.

Da Silva, F. (2014). Sentido personal, significado social y actividad de estudio: una revisión teoría. Psicol. Esc. Educ, 18 (2): 265-272. 


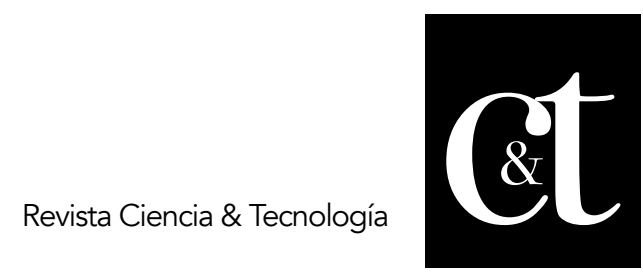

No. 28, 31 de octubre de 2020

ISSN impreso: 1390 - 6321

ISSN online: 2661 - 6734

Covey, S. (1989). Los siete hábitos de la gente altamente efectiva. Buenos Aires: Editorial Free Prees

Gil, J.; Morales, M.; Chou, R. (2018). La actividad de estudio y el proceso de asimilación consciente de los conocimientos. ¿Están preparados los estudiantes universitarios? Revista Conrado, 14 (62).

González, F. (1989). La personalidad, su diagnóstico y desarrollo. La Habana: Pueblo y Educación.

González, J.; García, A. y Reyes, R. (2016). La Cultura de participación de la familia en los proyectos de vida profesional de los niños, adolescentes y jóvenes. Revista Universidad y Sociedad, 8 (2), 72-78.

Pérez, R. (2013). Fundamentos de Psicología. La Habana: Pueblo y Educación.

Torroella, G. (2002). Cómo estudiar con eficacia. La Habana: Pueblo y Educación.

Real Academia de la Lengua Española (2001). Diccionario de la Lengua Española. Disponible en https://www.rae.es/recursos/diccionarios/diccionarios-anteriores1726-2001/diccionario-de-la-lengua-espanola-2001 